University of Nebraska - Lincoln

DigitalCommons@University of Nebraska - Lincoln

Papers in the Earth and Atmospheric Sciences

Earth and Atmospheric Sciences, Department

1986

\title{
Identification and Analysis of Climatic Fields through Dot-Density Shading
}

Stephen J. Lavin

University of Nebraska - Lincoln

Randall S. Cerveny

University of Nebraska - Lincoln, cerveny@asu.edu

Follow this and additional works at: https://digitalcommons.unl.edu/geosciencefacpub

Part of the Earth Sciences Commons

Lavin, Stephen J. and Cerveny, Randall S., "Identification and Analysis of Climatic Fields through DotDensity Shading" (1986). Papers in the Earth and Atmospheric Sciences. 598.

https://digitalcommons.unl.edu/geosciencefacpub/598

This Article is brought to you for free and open access by the Earth and Atmospheric Sciences, Department of at DigitalCommons@University of Nebraska - Lincoln. It has been accepted for inclusion in Papers in the Earth and Atmospheric Sciences by an authorized administrator of DigitalCommons@University of Nebraska - Lincoln. 


\title{
Identification and Analysis of Climatic Fields through Dot-Density Shading
}

\author{
StePHEN J. LAVIN AND RANDALl S. CeRvenY \\ Department of Geography, University of Nebraska-Lincoln, Lincoln, NE 68588
}

9 October 1985 and 18 February 1986

ABSTRACT

\begin{abstract}
Gridded matrices of climatic data can be mapped by computer with a variety of symbolization methods, all of which have some shortcomings. A new mapping procedure, dot-density shading, is herein proposed as an alternative mapping form. Dot-density shading produces continuous-appearing dot patterns whose density is proportional to the data. Significant algorithms are those for computing dot numerosity and quasi-random dot placement. A number of applications, including data error detection and anomaly recognition are discussed.
\end{abstract}

\section{Introduction}

Development and refinement of numerical modeling and data collection techniques has led to the creation of large gridded data matrices. Global climate models, for example, often contain thousands of data points (Gates et al., 1971; Sellers, 1983). Large matrices, however, permit the introduction of a variety of errors at individual grid locations through faulty observations, mistyping or other inaccuracies. While mathematical methods, e.g., smoothing, may mask errors, such techniques may also minimize climatologically significant fluctuations. Direct cartographic analysis of initial data is one methodology that can detect many inaccuracies in datasets and distinguish them from real climatic extremes.

Cartographic analysis is also important in the detection of significant trends and anomalies in climatic data fields. Basic hemispheric maps of surface pressure, for example, have long been used in synoptic climatology to examine semipermanent pressure cells (Petterssen, 1958; Barry and Perry, 1973). Numerical modelers have also used this analysis; for example, Sellers (1983) identified real and anomalous precipitation areas in his climate model using the mapped output.

Detection of climatic trends and anomalies through cartographic analysis requires the selection of an appropriate mapping technique. Figure 1 illustrates maps produced from three widely available automated mapping packages. All maps are made from the same data set, global precipitation for July (Schutz and Gates, 1972). Figure la was produced with the SYMAP mapping package (Fisher, 1975); Fig. $1 \mathrm{~b}$ is an isoline map generated with the SURFACE II graphics software (Sampson, 1978); and Fig. 1c, a perspective block diagram, was also made by SURFACE II. Although se- lection of these forms of symbology is clearly logical in climatic mapping, each has significant shortcomings.

The detection of large errors and anomalies using the mapping techniques shown in Fig. 1 is possible, but smaller-scale phenomena may be entirely missed. On the SYMAP and SURFACE II isoline maps in Figs. $1 a, b$, discrepancies may not be detected unless the appropriate isoline interval is chosen. If one is not aware in advance of map construction that anomalies may exist, selection of the appropriate interval becomes a matter of chance. The perspective diagram in Fig. 1c presents a different set of problems caused by presenting the truth, but not the whole truth. Although gradations in the surface are well represented, parts of the surface are always hidden. The researcher must be aware of the existence and locations of anomalies and salient trend elements before the map is made if the proper degree of surface rotation, viewing height (azimuth) and vertical scaling is to be selected. This, unfortunately, is not commonly the case and several versions of a map must be made in order to see the entire surface.

In view of the existence of these problems a new automated method for mapping climatic and meteorological data is herein proposed. The technique, referred to as dot-density shading, produces dot patterns whose density in a given location is proportional to the data value in that location. Figure 2 shows a map made by the dot-density shading computer program. This map shows the same data as was mapped in Fig. 1, global precipitation for July.

Dot-density maps have number of advantages over isoline maps and perspective diagrams:

1) Isoline maps symbolize data only along isolines. In order to understand the entire surface, the map user must fill in the gaps between isolines by visual inter- 

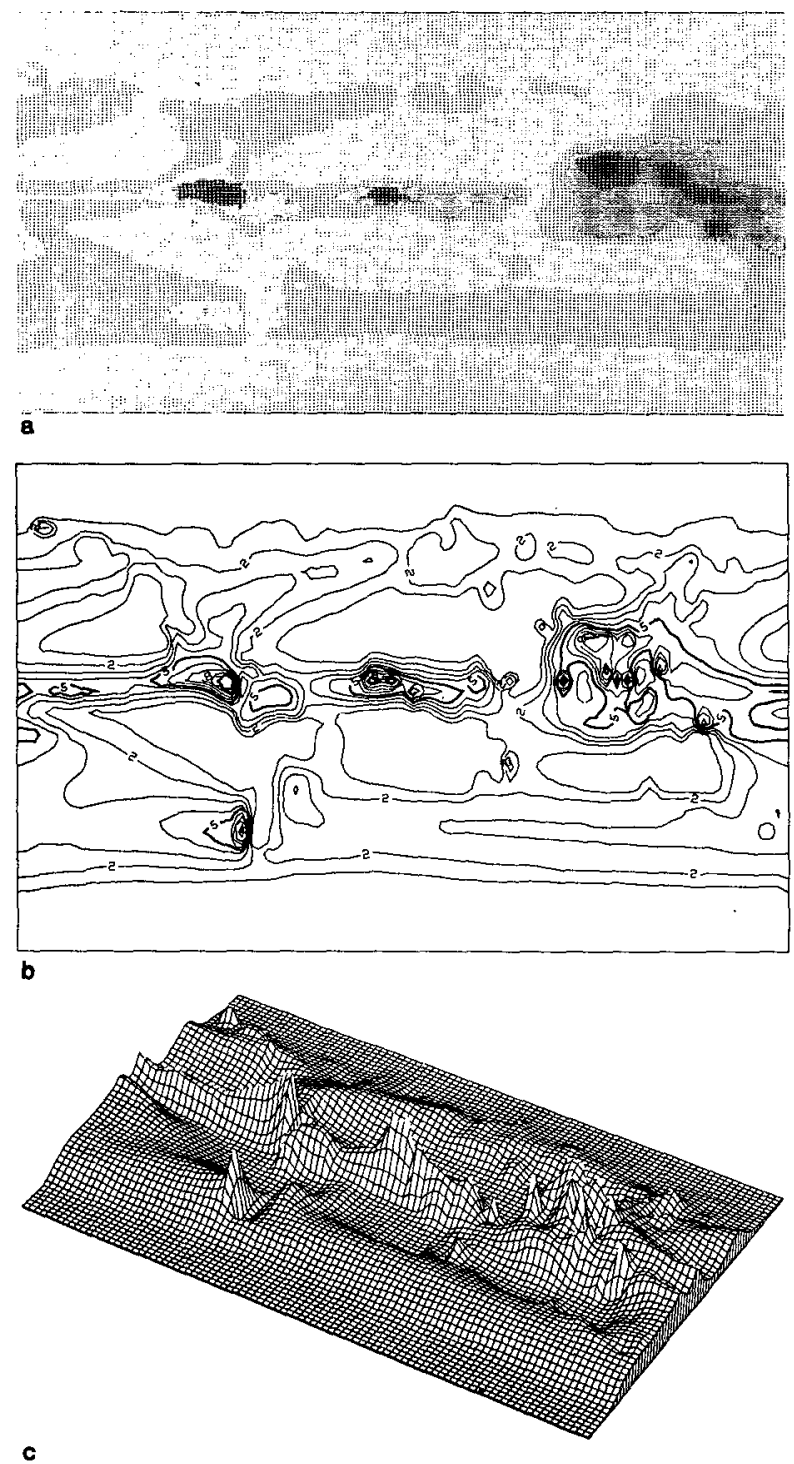

FiG. 1. Varieties of symbology for computer-generated climate maps of July precipitation. (a) SYMAP isoline map; (b) SURFACE II isoline map; (c) SURFACE II perspective diagram.

polation. This is a process which is particularly prone to estimation error (Griffin and Lock, 1979; Morrison, 1971; Hsu and Robinson, 1970).

2) Dot-density shading allows for virtually immediate detection and recognition of surface trends and anomalies in the distribution of a variable. A minimum of time and map reading skill are required to understand that darker patterns represent higher data values and lighter patterns depict lower data values. On the other hand, understanding isoline maps requires considerable skill and maps must be studied carefully.

3) Dot-density maps are two-dimensional planimetric presentations. Unlike perspective block diagrams, the entire surface of the map can be viewed at once. Consequently, there is no need to manipulate rotation angles, azimuths or vertical scaling.

4) Maps produced by dot-density shading are unitless, relative representations of data. For example, a map of precipitation would exhibit the same pattern regardless of the data being in millimeters or inches. Therefore, the symbolization is more universal in nature than is isoline mapping.

In subsequent sections of this paper, we discuss the mapping technique used in dot-density shading and examine a variety of applications of this technique.

\section{Dot-density shading methods}

A primary goal in dot-density shading is to use all available data, without further interpolation, in order to show the statistical surface in its entirety. In this way, the visual continuity of a surface and events that tend to disrupt continuity are depicted. A numerical distribution which changes gradually over space is shown with gradually changing dot-densities, and steep numerical gradients are mapped with abrupt density shifts. Density of dots is achieved by adjusting the number of dots plotted and the locations in which dots are plotted. These two controls are of singular importance in dot-density shading and require further discussion.

\section{a. Number of dots}

The number of dots in any given area of a map is proportional to the data value representing that area. Figure 3 shows how dot-density varies with data intensity. Between any two adjacent tic marks, dot density is constant; only between adjacent blocks does the density change. Nevertheless, the diagram gives the appearance of continuous changes in density. Additionally, information on the number of dots plotted in each block and the percentage of block area covered with data is provided in Fig. 3.

The computation for the number of dots $\left(N_{d}\right)$ is

$$
N_{d}=\left(P / A_{d}\right) A_{g}
$$

where $P$ is the desired proportion of the area covered with dots, $A_{d}$ is the area of a single dot, and $A_{g}$ is the area of the plotting unit (in this case, a square grid cell). The proportion of the area covered with dots, $P$, is determined by linear scaling, so that dot density is proportional to data. The maps in this paper are scaled between $P=0.0$ (zero dot density) and $P=0.8(80 \%$ of the plotting unit area is covered with dots) using the following equation:

$$
P=\left(\mathrm{X}_{i}-\mathrm{X}_{\min }\right)\left(P_{\max }-P_{\min }\right) /\left(X_{\max }-X_{\min }\right)
$$

where $X_{i}$ is the data value in a plotting unit, $X_{\min }$ is the smallest data value in the dataset, $P_{\max }$ is the largest 


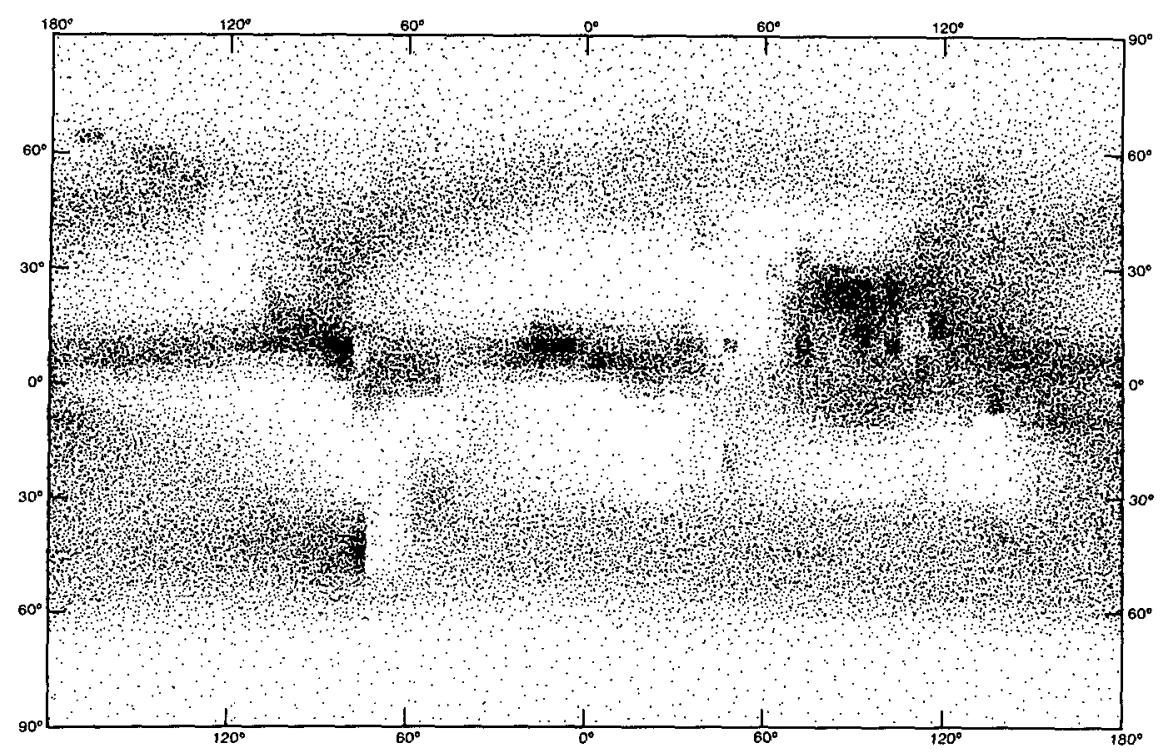

FIG. 2. A map of July precipitation produced with dot-density shading.

desired proportion of area covered by dots, $P_{\min }$ is the lowest desired proportion of area covered by dots, and $X_{\max }$ is the largest data value.

\section{b. Determination of plotting locations for dots}

Although constructing maps with dots is by no means a new idea, previous attempts have placed dots in totally random locations (Dutton, 1978). As a consequence, dots overlap and the accuracy of $P$ is diminished.

Dot-density shading uses a different strategy for dot placement, referred to as quasi-random dot placement. The quasi-random procedure is designed to assure that no dots can overlap and thus preserves the accuracy of $P$. The procedure for plotting dots is explained in the following four steps:

1) Dot-density shading begins with the input of a regular square grid of data values, similar to that produced in many first-stage interpolation algorithms used for isoline mapping.

2) Square grid cells are constructed around each node of the gridded data matrix. These cells become the primary plotting units in subsequent operations, although the cells themselves are never plotted. Next, the number of dots, $N_{d}$, is computed for the data value in each cell, as in (2.1).

3) Cells are partitioned into rectangular subcells, with one cell for each dot required. Therefore, the number of subcells is equal to the number of dots.

4) One random cartesian coordinate is generated in each subcell and a dot is plotted at that coordinate. The computation of coordinates includes a constraint for plotting each individual dot entirely within the bounds of its parent subcell. In actuality, some dot overlap may occur depending upon the precision of arithmetic computations and the accuracy of the plotting device.

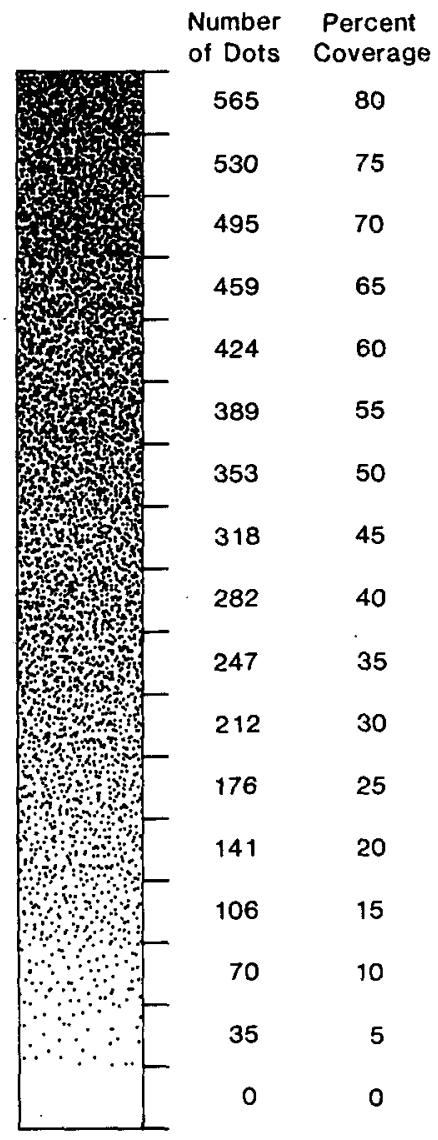

FIG. 3. Visual effects of the number of dots in quasi-random dot plotting. Dot density is proportional to data intensity. 


\section{Climatic applications of dot-density shading}

Specific applications of mapping algorithms in climate research can define the strengths and weaknesses of a given cartographic technique. Isolining, for example, is often successfully employed in identification and location of areas of maximum and minimum values. This technique, however, is not often used in investigation of the interrelationships that exist between different regions.

Dot-density shading can be used effectively in many ways that are distinctly different from uses of other mapping techniques. It therefore expands the variety of uses in which cartographic analyses can be employed in climatology. In this section, we will briefly examine specific applications of dot-density shading and discuss the performance of the technique. These applications include recognition of errors in initial data, errors amplified through computational instabilities, and identification of significant climatic features.

\section{a. Recognition of initial data errors and errors ampli- fied through computational instabilities}

A constant source of potential error in numerical modeling is data entry. Identification of erroneous initial data is a task particularly well suited for cartographic analysis. A dataset of grid point elevations provides a useful example of information required in initializing most models. We have used the topography dataset created for version of the Mintz-Arakawa General Circulation Model (Gates et al., 1971). This dataset expresses elevation in hundreds of feet for a global grid network having $4^{\circ}$ lat by $5^{\circ}$ long intervals.

Various errors were introduced into the dataset to evaluate the effectiveness of dot-density shading in detecting those errors (Fig. 4). Changes in the elevation dataset were introduced in northeast South America where a value of 90 hundred feet ( 27432 meters) was inserted rather than the correct elevation of 9 hundred feet (2743.2 meters). This demonstrates a common type of error where a value is mistyped in an adjacent column of the input file. A similar type of error is visible in Australia. As seen in Fig. 4, errors are easily recognizable through dot-density shading.

A common type of data entry error is the transposition of numbers. In Fig. 4, elevations were altered in the northeastern Himalayas $\left(46^{\circ} \mathrm{N} 100^{\circ}-105^{\circ} \mathrm{E}\right)$. At $46^{\circ} \mathrm{N}, 105^{\circ} \mathrm{E}$, the elevation of $5300 \mathrm{ft}(1615 \mathrm{~m})$ was transposed to $3500(1067 \mathrm{~m})$. This is an example of an error that is clearly displayed using a dot-density shading.

Many errors involving initial data are not traceable to the interpolation of values into data-poor regions or through similar empirical limitations. While these errors are usually not immediately detectable, they can be identified in a time series of maps as they amplify. To study time series, error recognition we have employed an experimental global climate model developed at the University of Nebraska. Because we are primarily interested in demonstrating the dot-density shading technique, characteristics of the model will not be discussed in detail except to state that it is a monthly averaged energy balance model capable of producing a $500 \mathrm{mb}$ circulation. The model was "seeded" with an initial error (that amplified over time) in the 500 mb zonal wind speeds. Dot-density maps of the January, March and May $500 \mathrm{mb}$ zonal wind speeds (Figs. $5 a, b, c)$ were created to demonstrate the visualimpact of this instability.

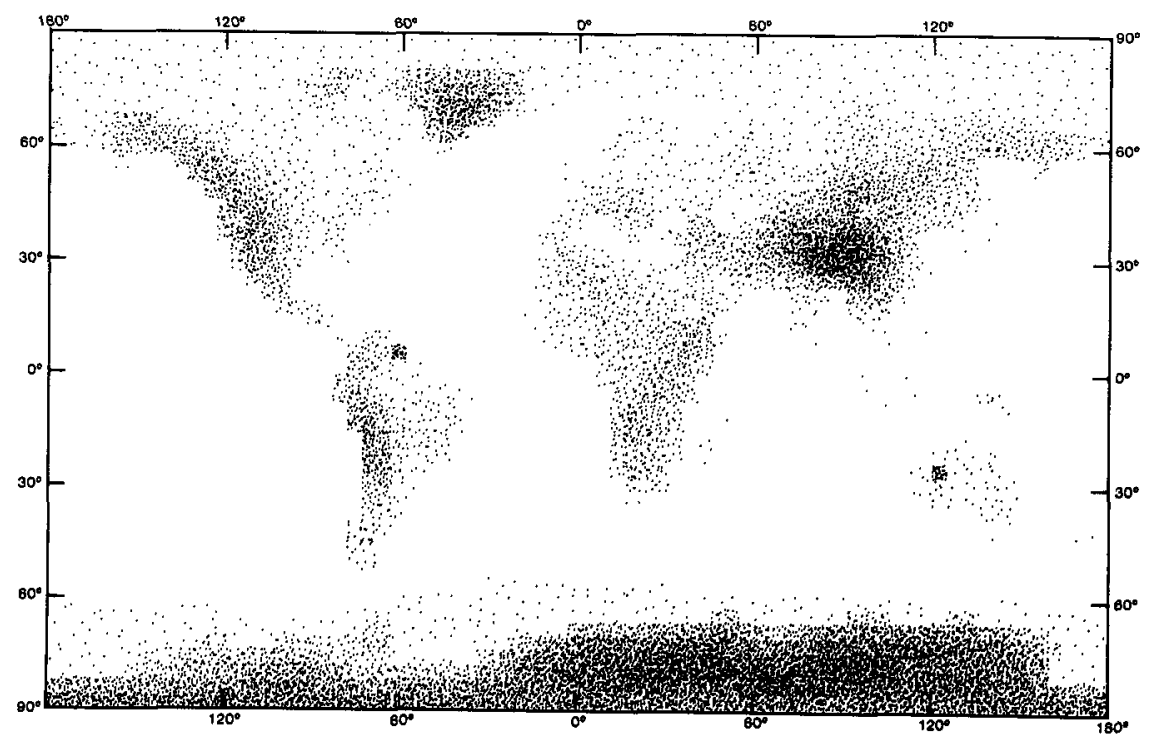

FIG. 4. Dot-density shading map of global topography with manually entered errors. 

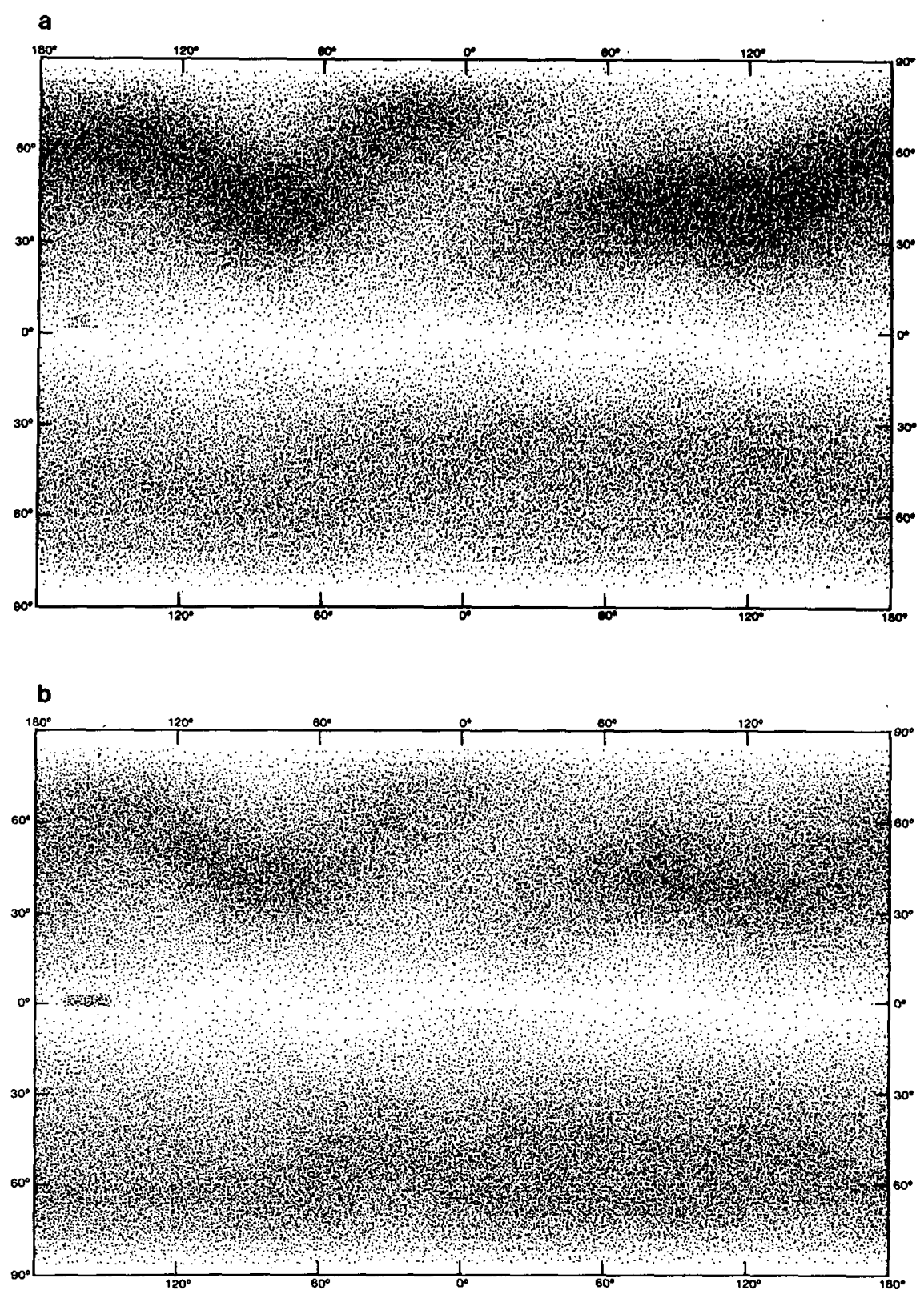

FIG. 5. Time series of $500 \mathrm{mb}$ zonal wind speeds generated from an experimental global climate model. Amplifying error is inserted in the equatorial Pacific Ocean at $2^{\circ} \mathrm{N}, 165^{\circ} \mathrm{W}$. (a) January zonal winds; (b) March zonal winds; (c) May zonal winds.

A small perturbation in the $500 \mathrm{mb}$ flow might be discerned in the January map in the Pacific equatorial region $\left(2^{\circ} \mathrm{N}, 165^{\circ} \mathrm{W}\right)$. However, by March, the fluctuation has increased in both magnitude and longitudinal extent and becomes easily detectable against the backdrop of weak winds of the intertropical convergence zone (ITCZ). In May, the ITCZ has moved into the Northern Hemisphere and the amplifying error becomes a significant phenomenon. Additionally, the perturbation's western "tail" becomes more evident as the easterly flow of the ITCZ increases through time in the affected region.

\section{b. Identification of significant climatic features}

Mapping techniques in climatology have been used for the detection and interpretation of features and patterns associated with climatic variables. Modeling research, for example, often contains visual comparisons of simulated and observed distribution of the same variable (e.g., Gates, 1972; Schlesinger and Gates, 1980; Sellers, 1983; Washington and Meehl, 1984). Similarly, empirical observations are frequently discussed with the assistance of mapping techniques (e.g., Budyko, 1974; Lamb, 1977). 


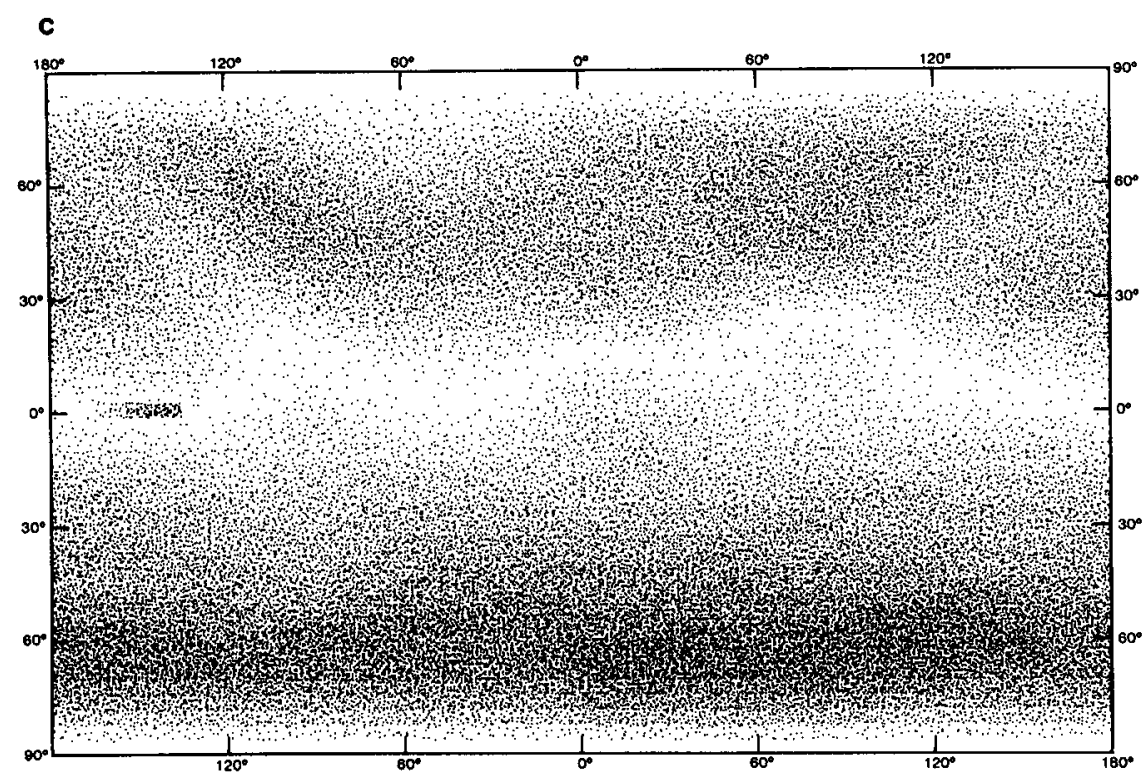

FIG. 5. (Continued)

Reexamination of Fig. 2, the July precipitation pattern for the globe (Schutz and Gates, 1972), provides a basis to evaluate the effectiveness of dot-density shading in identifying climatic features and patterns. The most dramatic features are the southeast Asian monsoon and the ITCZ indicated by heavy precipitation near $4^{\circ} \mathrm{N}$. The large subtropical high pressure cells are easily identified in the North and South hemispheres by the lack of precipitation occurring near $30^{\circ}$ lat. A broad belt of precipitation stretching from the southeast United States northeastward into Europe is clearly seen in the dot-density map while the isohyet map (Fig. 1b) shows a less interpretable, disjointed pattern.

In addition to specific features, large regions can be easily seen with dot-density shading. Besides the ITCZ, regions under the influence of subtropical high pressure and regions experiencing cyclonic activity in the band of the westerlies in both hemispheres are easily detectable. Monsoonal precipitation is more recognizable as a single, but complex, feature in the dot-density product than in an isohyet map of the same field.

Similarly, the dot-density representations of the 500 mb zonal winds (Figs. 5a, b, c) can be used as examples of employing this technique in the identification of climatic features. Zonal wind velocities (in meters per second) are more continuous than precipitation data and create a smoother pattern than that associated with precipitation. A strong Rossby wave oscillation is easily visible in the $500 \mathrm{mb}$ flow for the Northern Hemisphere in January. This pattern becomes more zonal and less intense as the year progresses. In contrast, the more oceanic Southern Hemisphere has a very broad zonal pattern that amplifies dramatically as the Southern
Hemisphere's winter advances. Separating the two hemispheres is a distinctive belt of low wind speeds in the location of the ITCZ. Isolining of upper-level wind fields often creates maps that have less recognizable patterns and are hard to interpret (e.g., Schutz and Gates, 1972).

\section{Conclusions}

Our goal in this paper has been two-fold: the enlargement of the climatologists' arsenal of available mapping methods by proposing a new technique for symbolizing gridded data, and the proposal of dot-density shading as a remedy to some of the shortcomings of other map symbols. We suggest that in the analysis of trends and anomalies, it may be advisable to use a variety of complementary symbolizations in order to develop the most complete possible conceptualization of a surface. In this regard, automated mapping methods allow a single gridded data file to be mapped in either isoline, perspective block or dot-density shading forms. Additionally, photomechanical methods permit the combination of maps, i.e., photographically printing both isoline and dot-density distributions together in overlay format.

In some respects, cartographic display and analysis of climatic information has been bound by adherence to the tradition of isoline use. While there are good reasons for following that tradition, there are also logical reasons for using alternative map symbols. Dotdensity shading is one such alternative.

\section{REFERENCES}

Barry, R. G., and A. H. Perry, 1973: Synoptic Climatology. Methuen, 555 pp. 
Budyko, M. I., 1974: Climate and Life. Academic Press, 508 pp.

Dutton, G., 1978: Experiments in contouring and shading: A DOT. MAP portfolio. Laboratory for Computer Graphics and Spatial Analysis, Harvard University, $63 \mathrm{pp}$.

Fisher, H. T., 1975: SYMAP. Laboratory for Computer Graphics and Spatial Analysis, Harvard University. [Software package.]

Gates, W. L., 1972: The January global climate simulated by the two-level Mintz-Arakawa model: A comparison with observations. R-1005-ARPA, The Rand Corp., Santa Monica, 107 pp.

- E. S. Batten, A. B. Kahle and A. B. Nelson, 1971: A documentation of the Mintz-Arakawa two-level atmospheric general circulation model. R-877-ARPA, The Rand Corp., Santa Monica, $408 \mathrm{pp}$.

Griffin, T. L. C., and B. F. Lock, 1979: The perceptual problem in contour interpretation. Cartogr. J., 16, 61-71.

Hsu, M. L., and A. H. Robinson, 1970: The Fidelity of Isopleth Maps: An Experimental Study. University of Minnesota Press, $57 \mathrm{pp}$.

Lamb, H. H., 1977: Climate: Present, Past and Future. Vol. 2, Methuen, $835 \mathrm{pp}$.

Morrison, J. M., 1971: Method-produced error in isarithmic mapping.
Tech. Monograph No. CA-5, American Congress on Surveying and Mapping, $76 \mathrm{pp}$.

Petterssen, S., 1958: Weather Analysis and Forecasting. Vol. 2, McGraw-Hill, 266 pp.

Robinson, A. H., R. D. Sale and J. L. Morrison, 1978: Elements of Cartography. 4th ed., Wiley and Sons, $448 \mathrm{pp}$.

Sampson, R. J., 1978: SURFACE II Graphics System. Kansas Geological Survey, $240 \mathrm{pp}$.

Schlesinger, M. E., and W. L. Gates, 1980: The January and July performance of the OSU two-level atmospheric general circulation model. J. Atmos. Sci., 37, 1914-1943.

Schutz, C., and W. L. Gates, 1972: Global climatic data for surface, 800mb, 400mb: July. R-1029-ARPA, The Rand Corp., Santa Monica, $180 \mathrm{pp}$.

Sellers, W. D., 1983: A quasi-three-dimensional climate model. $J$. Climate Appl. Meteor., 22, 1557-1574.

Trewartha, G. T., 1981: The Earth's Problem Climates. 2nd ed., University of Wisconsin Press, $371 \mathrm{pp}$.

Washington, W. M., and G. A. Meehl, 1984: Seasonal cycle experiment on the climate sensitivity due to a doubling of $\mathrm{CO}_{2}$ with an atmospheric general circulation model coupled to a simple mixed-layer ocean model. J. Geophys. Res., 89, 9475-9503. 